\title{
Generation of charged droplets by field ionization of liquid helium
}

\author{
Chun-Cheng Tsao†, Julio D Lobo $\dagger$, Mitchio Okumura $\nmid$ and \\ Shui-Yin Lo $\ddagger \S$
}

$\dagger$ Arthur Amos Noyes Laboratory of Chemical Physics,

California Institute of Technology, Pasadena, CA 91125, USA

$\ddagger$ American Technologies Group Inc., 1017 Mountain Avenue, Monrovia, CA 91016, USA

Received 27 October 1997

\begin{abstract}
Positively charged helium droplets were produced by ionization of liquid helium in an electrostatic spraying experiment, in which fluid emerging from a thin glass capillary was ionized by applying a high voltage to a needle inside the capillary. At $2.2 \mathrm{~K}$, fine droplets $(<10 \mu \mathrm{m}$ in diameter) were produced in pulsed sprays or showers with total currents as high as $0.4 \mu \mathrm{A}$ at relatively low voltages (2-4 kV). Ionization was accompanied by a visible glow at the needle and glass tips. Droplet formation was suppressed at $3.5 \mathrm{~K}$. In contrast, liquid nitrogen formed a well-defined Taylor cone with droplets having diameters comparable to the jet $(\approx 100 \mu \mathrm{m})$ at much lower currents $(3 \mathrm{nA})$ and higher voltages $(9 \mathrm{kV})$, in agreement with previous results. The mechanism for charging in these liquids was proposed to be field ionization, identical to the processes leading to conduction in cryogenic insulating liquids observed by Gomer. The high currents resulting from field ionization in helium, together with the intrinsically low surface tension of helium I, led to charge densities that greatly exceeded the Rayleigh limit, thus preventing formation of a Taylor cone and resulting in Coulomb explosion of the liquid.
\end{abstract}

\section{Introduction}

Clusters and droplets of helium are expected to exhibit novel physics analogous to that observed in the liquid. Charges injected into liquid helium have served as unique probes of elementary excitations in the superfluid. Similarly, charges may provide a method for elucidating the quantum mechanical and coherent properties of helium clusters and droplets. Multi-charged helium droplets [1] are in some ways analogues of multi-electron bubbles [2] in liquid helium. There have been some theoretical predictions of their properties, but these droplets are not well characterized experimentally. The purpose of the present work is to develop a method for generating a beam of multi-charged helium clusters and droplets. Such a beam could then be applied to study the physics of these particles.

Helium clusters can be produced directly in the vapour phase. In earlier experiments, singly charged species were formed by nucleation around charges introduced into cold vapour [3,4]. In several recent experimental studies, charged helium clusters have also been produced by generating neutral clusters in a supersonic expansion followed by ionization of the gas phase clusters. Varying the stagnation pressure and temperature behind the nozzle controls the size of the neutral helium clusters. Several

$\S$ Visiting Associate, Department of Chemistry, California Institute of Technology, USA. methods of ionizing these clusters have been reported: electron bombardment $[5,6]$, electron attachment [7] and, most recently, synchrotron radiation [8,9]. Although the supersonic expansion techniques have proved to be very reliable for forming singly charged clusters, multi-charged species possessing more than a few elementary charges have not been reported. Furthermore, the ionic clusters tend to be very small, because the weakly bound van der Waals clusters fragment extensively upon ionization.

Larger multi-charged droplets have been extracted directly from charged liquid helium surfaces by a Rayleigh-Taylor instability mechanism. Boyle and Dahm [10] introduced high charge densities into liquid helium films with a $\beta$-emitting source and extracted droplets into the gas phase by appliance of an electrical field. They reported the formation of gas phase droplets of radius $r=0.52 \mathrm{~mm}$ and charge $q \simeq 10^{-11} \mathrm{C}$. The experiment of Boyle and Dahm was conducted in the temperature range $3.24 \mathrm{~K}<T<5.13 \mathrm{~K}$, above the $\Lambda$ point. Volodin and Khaikin [11] extended that work by characterizing the surface discharge in superfluid helium at $1.2 \mathrm{~K}$. They measured a threshold of $1900 \mathrm{~V} \mathrm{~cm}^{-1}$ above which surface instabilities led to the ejection of liquid jets.

Lo has proposed that multiply charged superfluid helium clusters could be generated by field ionization or field emission of liquid helium II. In this approach, the liquid is charged by applying a high voltage to a sharp 


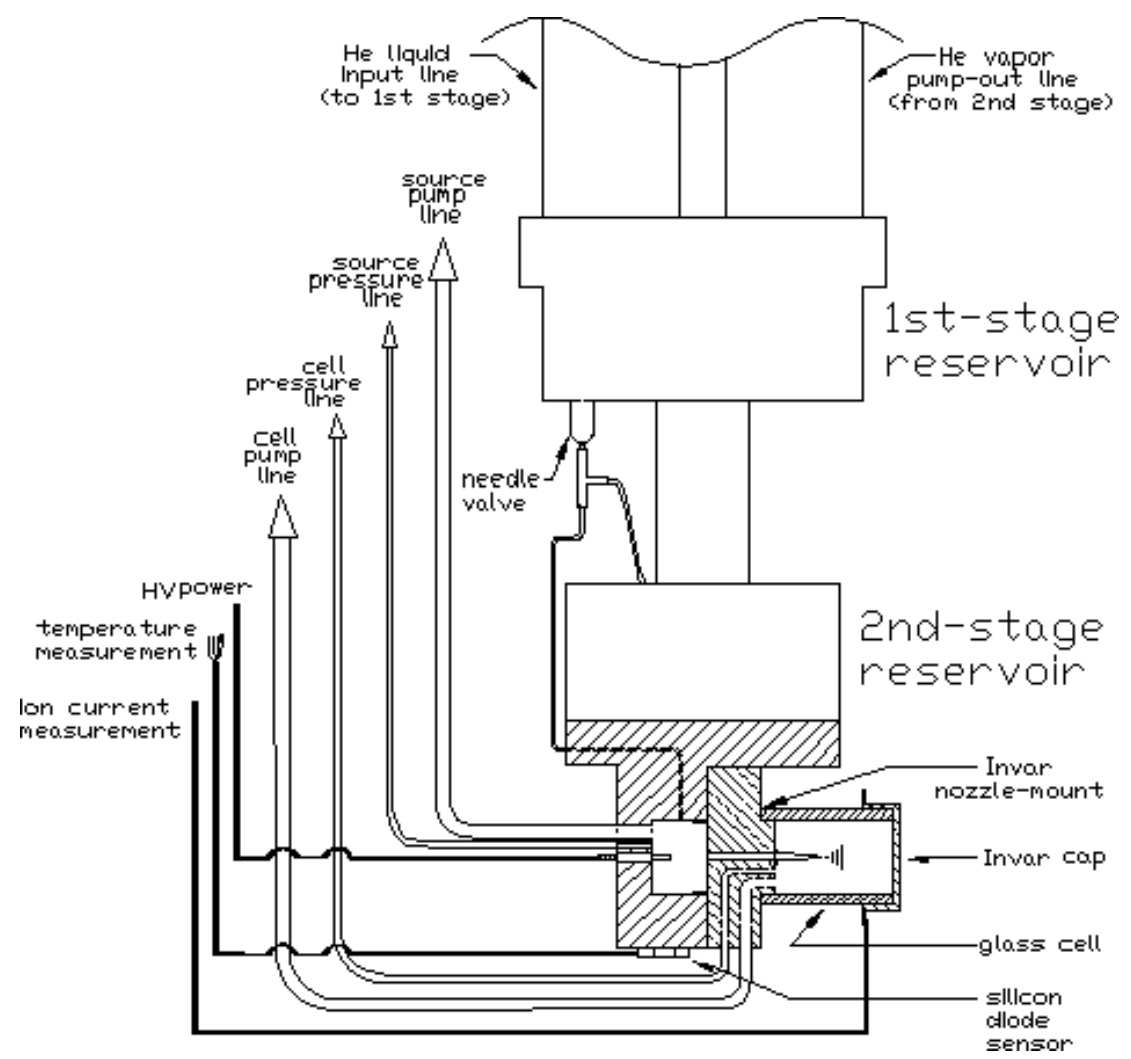

Figure 1. A detailed schematic diagram of the source, droplet cell and cryostat.

tip in the liquid and is then extracted through a nozzle into a vacuum chamber. Field ionization and emission have been elucidated in bulk liquid helium [12,13] and in liquid helium films [14]. A similar approach, electrostatic spraying, has been applied by Turnbull and co-workers [15] to form charged droplets of other cryogenic liquids such as hydrogen [16, 17] and nitrogen [18].

Of these methods, field ionization charging and electrostatic spray formation of droplets has the most promise for generating beams of multi-charged helium droplets. Direct ionization of liquid helium will yield clusters that are significantly larger and possess higher charge states than clusters formed by ionization of supersonic expansions, while the electrostatic spray methods should generate currents that are orders of magnitude higher than those possible with $\beta$-ray emitting sources.

In this paper we describe the generation of multicharged positive liquid helium droplets by field ionization and electrostatic spraying. We discuss the mechanism for droplet formation and compare the observed behaviour with that of droplet formation with liquid nitrogen.

\section{Experimental details}

\subsection{The apparatus}

2.1.1. Overview. The apparatus consisted of a cryogenically cooled electrostatic spraying source (figure 1). The source was assembled at the bottom of a modified optical cryostat, which could maintain the source at temperatures as low as $1.8 \mathrm{~K}$. The source was composed of three major parts: the source body, which contained the liquid reservoir, a glass capillary and a glass droplet cell. Liquid flowed from the reservoir through the capillary and into the droplet cell. The liquid was ionized inside the capillary by a tungsten needle positioned with its tip slightly recessed from the nozzle. Droplets were ejected from the nozzle into the droplet cell, a glass cylinder sealed to the outer face of the nozzle mount. A metal cap on the opposite end of the glass tube served to seal the droplet cell and to function as a Faraday cup. Droplets formed in the cell were viewed through the glass by a camera coupled to a microscope.

2.1.2. The cryostat. The source was attached to the bottom of a two-stage RMC model CT-610-1 liquid-helium cryostat. The $4.2 \mathrm{~K}$ first-stage of the cryostat was designed to be continuously fed with liquid helium. The second stage was a ${ }^{4} \mathrm{He}$ pot that could be pumped to achieve $1.5 \mathrm{~K}$. The stages were connected through a needle valve, which was designed to regulate the liquid flow. After the needle valve, the tubing was split into two lines, one to the second stage and another to the source.

Three layers of radiation shields surrounded the source to minimize radiative heating. The second and outer shields had glass windows to allow viewing of the source, while blocking some IR radiation. The cryostat was kept inside a diffusion-pumped vacuum chamber maintained at pressures below $10^{-7}$ Torr. 


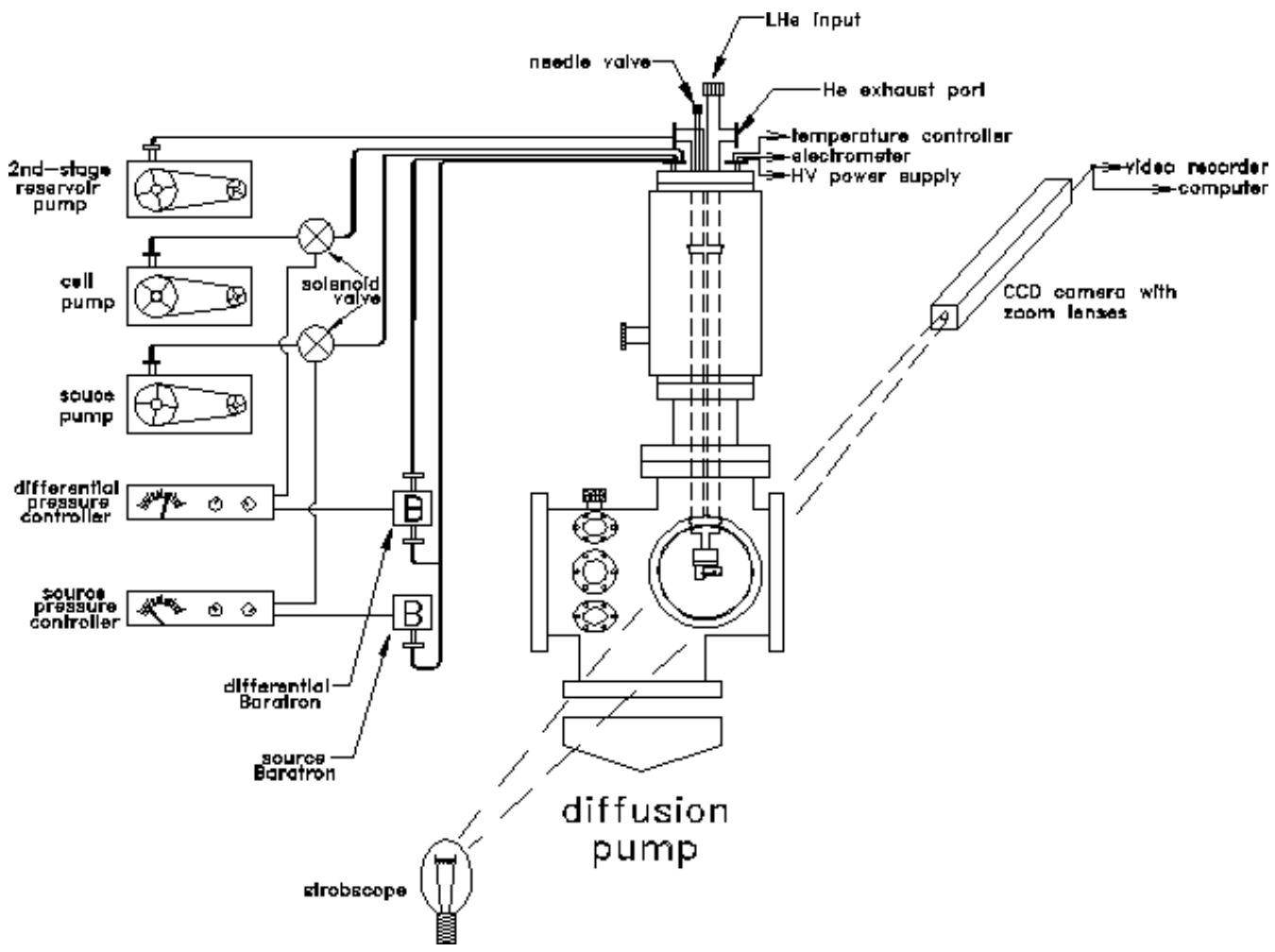

Figure 2. A schematic diagram of the apparatus.

2.1.3. The source. The source body consisted of a cylindrical disc $(3.8 \mathrm{~cm}$ in diameter) and a block of dimensions $2.54 \mathrm{~cm} \times 2.54 \mathrm{~cm} \times 1.14 \mathrm{~cm}$ machined from a single piece of OFHC copper. The source was mounted onto the helium pot with the surface of the cylindrical disc flush with the bottom of the pot. The source chamber consisted of a $1.475 \mathrm{~cm}$ diameter hole bored $5 \mathrm{~mm}$ deep into the front face of the block. On the back of the source block there were a high-voltage feed through and two $1.59 \mathrm{~mm}$ in diameter stainless steel tubes, one a pump-out line and the other a pressure-sensing line. Liquid cryogen was fed from the first stage of the cryostat through a stainless-steel tube (1.59 $\mathrm{mm}$ inner diameter) into the source chamber through an inlet opening on the disc.

The source chamber was capped with an Invar nozzle mount, which was sealed to the source body with an indium seal. The nozzle mount had two holes connecting the front face of the nozzle mount to the side of the piece, for the pumping and pressure-sensing lines. In the centre of the nozzle mount there was a hole for the glass nozzle, which was sealed to the nozzle mount piece with epoxy resin. The glass nozzle was formed by heating a glass capillary with a flame, stretching it and then, after cooling, breaking the tip at the desired inner diameter of $60-80 \mu \mathrm{m}$. The tungsten needle $(d=50 \mu \mathrm{m})$ with a tip of radius $r \approx 0.4 \mu \mathrm{m}$ was inserted into the capillary such that the tip was kept slightly $(0-100 \mu \mathrm{m})$ recessed from the orifice in order to prevent arcing.

2.1.4. The droplet cell. The droplet chamber consisted of a glass cylinder, of $13 \mathrm{~mm}$ inner diameter and $15.5 \mathrm{~mm}$ length. Both ends of the cylinder were sealed with indium gaskets. One end was sealed to the invar nozzle mount and the other to an Invar cap.

\subsection{Experimental procedures}

2.2.1. Overview. Figure 2 is a schematic diagram of the experiment. Liquid helium flowed from the source chamber, through the capillary and into the droplet cell. Both the source chamber and the droplet cell were pumped to maintain a pressure difference across the nozzle. As the liquid flowed through the nozzle, the electrical field due to the high potential of the needle charged the liquid. The charged droplets were sprayed into the droplet chamber, where they could be observed with a microscope. The total current was measured on a Faraday cup.

2.2.2. Temperature control. The first stage of the continuous-flow cryostat was maintained at $4.2 \mathrm{~K}$. Liquid helium from the first stage was leaked through a flowregulating needle valve and then teed off to the second stage of the cryostat and to the source. On the back of the source, there was one pumping tube and one pressure-sensing tube, which were connected to a flow control valve followed by a mechanical pump and a MKS 626 absolute pressure transducer, respectively. By controlling the vapour pressure of helium inside the source, the temperature could be varied. The temperature of the source was monitored with a silicon diode in thermal contact with the source body. The diode readout was monitored through a LakeShore model 
DRC-91C temperature controller. The temperature reading could be verified with the vapour pressure measurement.

2.2.3. Flow control. Liquid flow from the source chamber to the droplet cell was controlled by regulating the pressure difference across the nozzle. There were two stainless steel tubes connected to the droplet cell for pumping and pressure measurement of the droplet chamber respectively. By adjusting the rates of pumping from the source and the droplet chamber independently, the pressure drop across the nozzle could be adjusted from negative to positive several hundred torr (at negative pressure, the droplet cell was at a higher pressure than the source). The differential pressure across the nozzle was measured with a MKS 233B differential baratron. A MKS 250D pressure-controlling unit controlled the difference in pressure between the source and droplet the chamber. The controlling unit drove a flow-controlling valve located on the cell pump-out line.

2.2.4. Field ionization. Field ionization was induced by raising the needle to a positive dc potential. Current from a dc power supply passed through a $60 \mathrm{M} \Omega$ current-limiting resistor and an ammeter, both in series with the needle. A $200 \mathrm{M} \Omega$ to $200 \mathrm{k} \Omega$ voltage divider was connected after the $60 \mathrm{M} \Omega$ ballast to monitor the discharge on an oscilloscope. The voltage could be raised as high as $12 \mathrm{kV}$, above which the connectors would arc.

A sharp tip was made by electrochemically etching tungsten wire $50 \mu \mathrm{m}$ in diameter. Scanning electron microscope (SEM) images (figure 3(a)) indicated that the needle had a radius of curvature of $0.4 \mu \mathrm{m}$ or less prior to use. The tip became dull after several hours of operation, as can be seen in the SEM image in figure 3(b). Higher voltages were generally required as tip wear increased. The needle was replaced after each run.

2.2.5. Measurements. The ion current impinging on the Faraday cup (Invar cap) was measured with a Keithley model 485 pico-ammeter. The sensitivity of the ion current measurement was of the order of $1 \mathrm{nA}$. The Invar cap could be raised to high voltages to verify charging by deflecting the charged particles.

The formation of helium droplets was recorded with a long-distance microscope (Titan Tool model TZOVA) and a CCD camera (Cohu model 4110) which provided spatial resolution of up to $2 \mu \mathrm{m}$. The droplet was backlit by an AMETEK (model 1967) strobe light, which could be operated at 1-30000 flashes per minute.

2.2.6. Liquid nitrogen experiments. The liquid nitrogen experiments were performed in the same apparatus using a similar procedure. With nitrogen as the coolant, the temperature ranged between 67 and $77 \mathrm{~K}$, depending on the pressure maintained in the source. Our objective was to provide a benchmark for comparing the liquid helium results with the electrostatic spraying behaviour of other cryogenic liquids observed previously. The experiment was similar to that of Woosley and Turnbull [18], except that in (a)

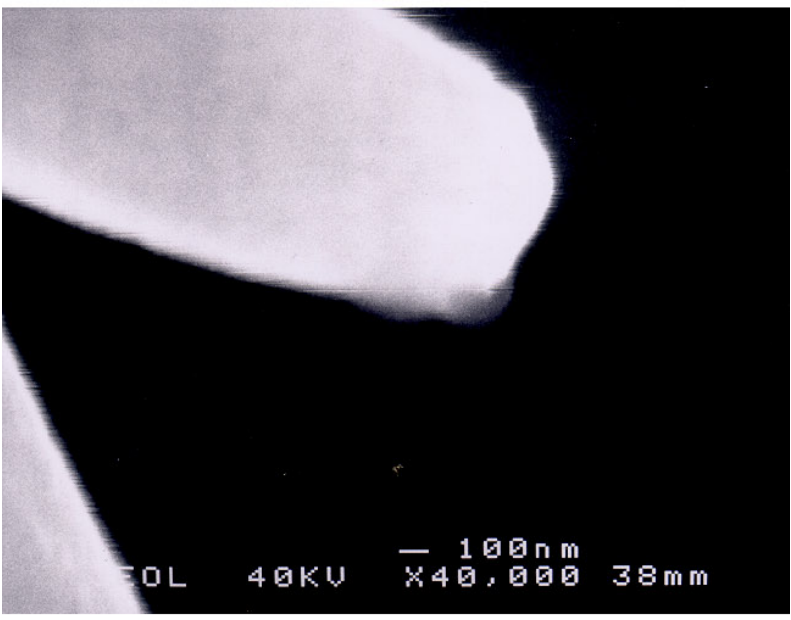

(b)

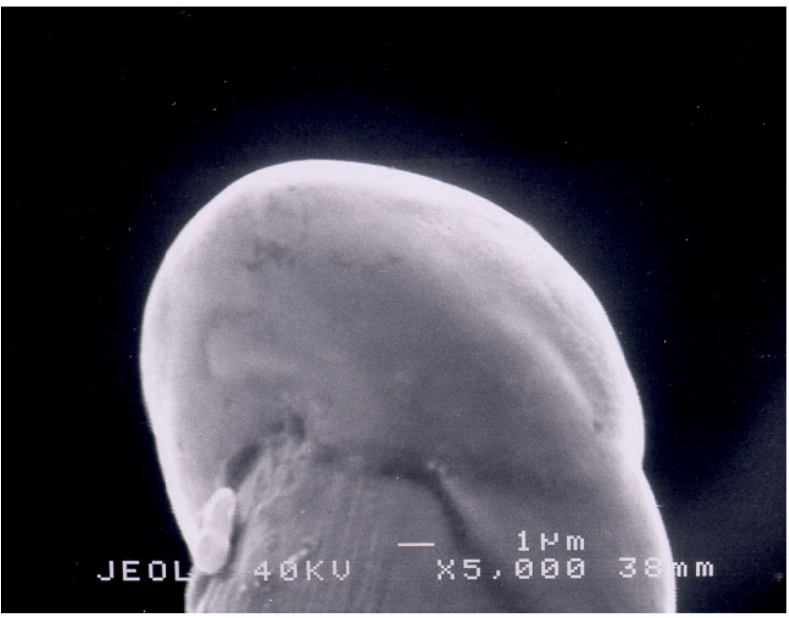

Figure 3. Scanning electron microscope images of typical tungsten tips. (a) An etched tip prior to use. The radius of the tip is approximately $0.4 \mu \mathrm{m}$. (b) A tip after a single run in liquid helium.

their apparatus the capillary and fluid flows were directed vertically downwards.

\section{Results}

\subsection{Liquid helium}

Three variables were changed independently to study the field ionization process: the source temperature, the pressure difference across the nozzle and the needle voltage. Ionization of liquid helium was characterized at three temperatures, 1.8, 2.2 and $3.5 \mathrm{~K}$. At each temperature, the pressure difference and voltage were independently varied to produce different spraying conditions. At $2.2 \mathrm{~K}$ two distinct modes of flow were observed, depending on the pressure difference across the nozzle. The measurements 
(a)

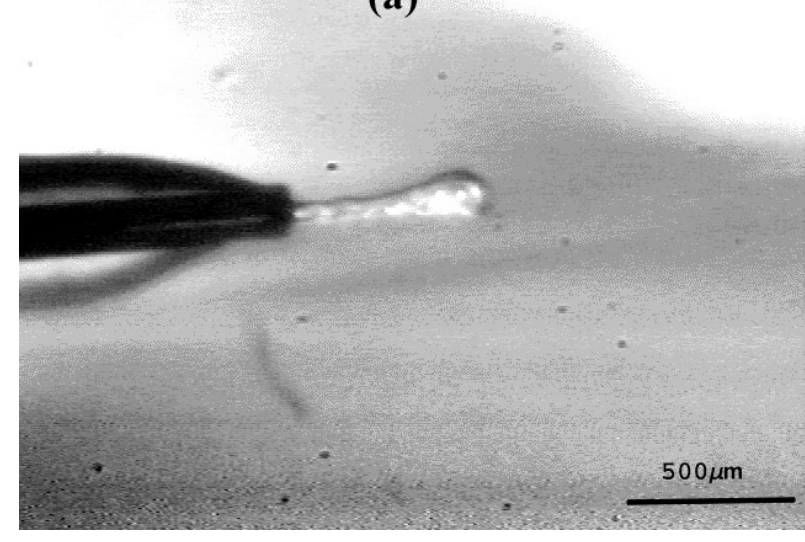

(b)

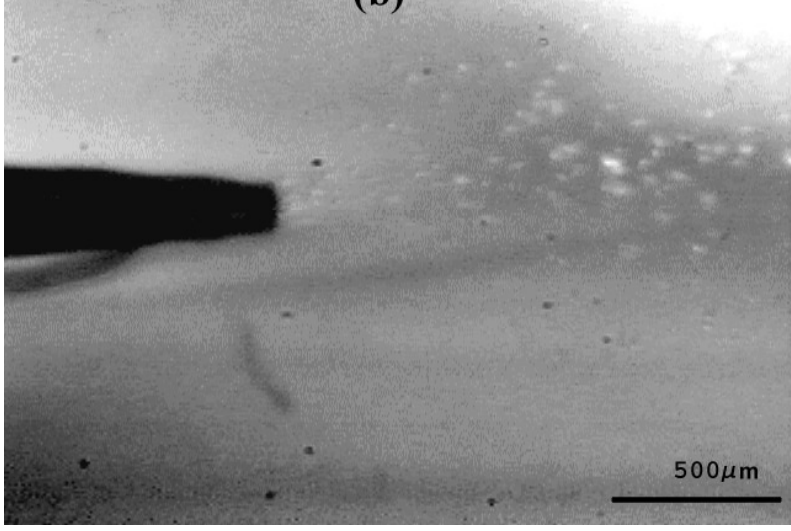

Figure 4. Liquid helium at $T=2.2 \mathrm{~K}$ and $\Delta P=2$ Torr. (a) No voltage applied to the needle tip. A drop clinging to the capillary is apparent. The burst of fluid emerging from the nozzle is induced by pressure fluctuations.

(b) $V=2 \mathrm{kV}$. A spray of fine charged droplets is observed. The drop on the capillary's exterior has shrunk.

at $3.5 \mathrm{~K}$ were intended to characterize the electrical discharge at higher temperatures. Measurements below the $\Lambda$ transition point of helium were performed at $1.8 \mathrm{~K}$.

3.1.1. Temperature 2.2 K. At $2.2 \mathrm{~K}$ with pressure differences across the nozzle of less than 2 Torr, liquid flowed slowly out of the nozzle and wetted the outside of the capillary. Large drops $(r>0.5 \mathrm{~mm})$ formed either on the tip or on the lower side of the capillary, as can be seen in figure 4(a). The drops occasionally burst due to fluctuations of the pressure difference, which were of the order of 0.5 Torr. Once voltage had been applied to the needle, the drop on the nozzle's tip grew. If the drop was hanging on the side of the capillary, it moved to the front of the capillary tip. Upon applying voltages of 2-4 kV (depending upon the needle's condition), the drop exploded into a shower of charged particles (figure 4(b)). Since pressure fluctuations ( $\geq 25 \%$ ) were relatively high, no data were acquired in this pressure-difference regime.

When the pressure difference across the nozzle was greater than 2 Torr, we observed a steady jet of liquid streaming out of the source (figure 5(a)). The application of
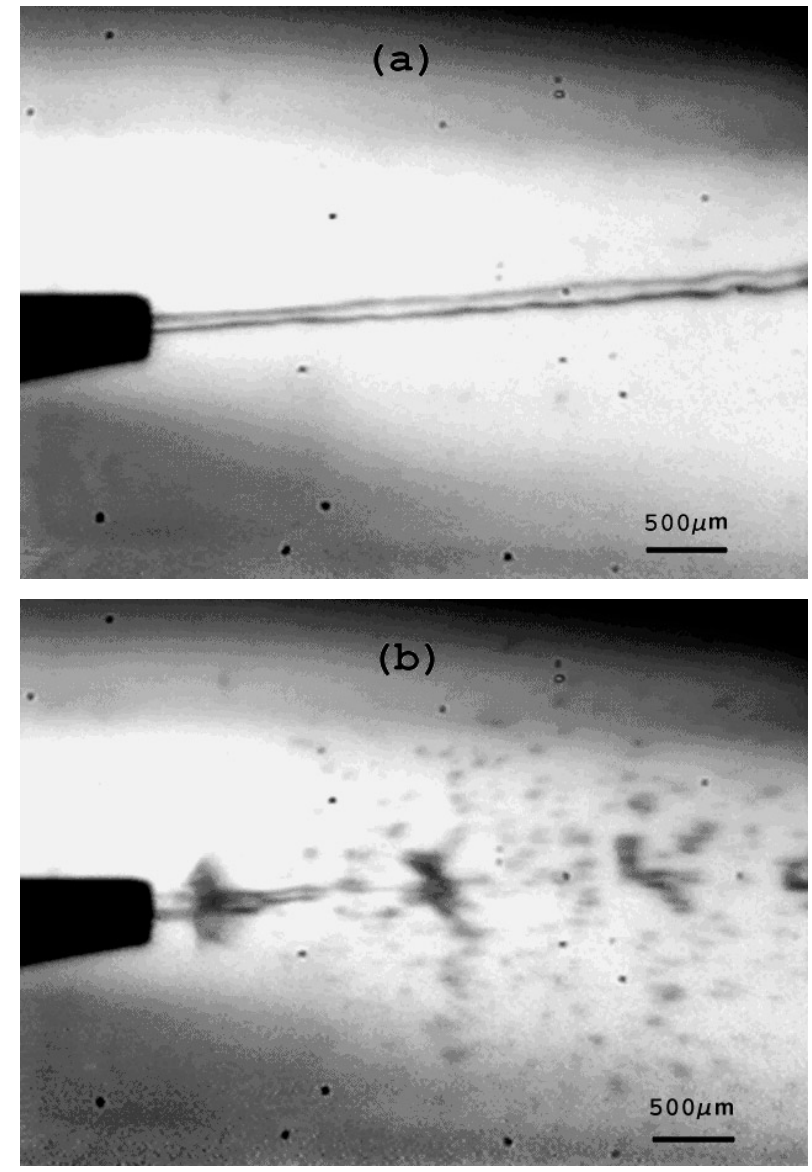

Figure 5. Liquid helium at $T=2.2 \mathrm{~K}$ and $\Delta P=5$ Torr. (a) No voltage applied to the needle. A stable jet with a diameter of $130 \mu \mathrm{m}$ emerges from the nozzle.

(b) $V=2 \mathrm{kV}$. A pulsed Coulomb explosion is apparent.

voltage to the needle did not affect the jet until a threshold had been reached. At the threshold, which ranged from 1.75 to $3.25 \mathrm{kV}$ depending on the condition of the needle's tip, a 'shower-like' explosion appeared in the jet (figure 5(b)) and about $100 \mathrm{nA}$ of ion current was detected on the Faraday cup. The explosion expanded radially from the centreline of the jet, as can be seen in figure 5(b). The smallest droplets that could be identified from the images were of the order of $1-10 \mu \mathrm{m}$ in diameter.

A weak, highly localized glow was observed on the needle's tip at these high currents, characteristic of a glow discharge. Parts of the glass nozzle were also found to glow. The weak glows on the glass were only seen when the room lights in the laboratory had been turned off. On further increasing the voltage about $1 \mathrm{kV}$ above the ionization threshold, arcing would occur. The highest stable ion current was about $400 \mathrm{nA}$. If the voltage was reduced after entering the glow regime, the high ion current remained until a cut-off of $0.5-1 \mathrm{kV}$ below the original threshold.

The liquid helium explosions in the glow regime were always pulsed, as can be seen in figure 5(b). We often observed several bursts in sequence within a single camera frame. The rate of pulsing increased as the voltage increased. However, no pulsing of the discharge voltage 


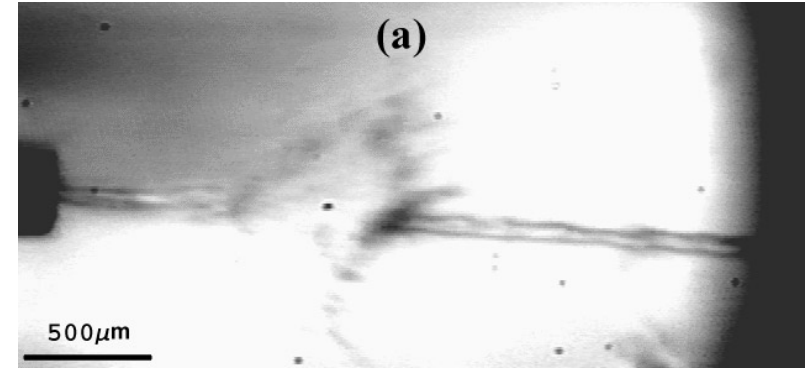

(b)
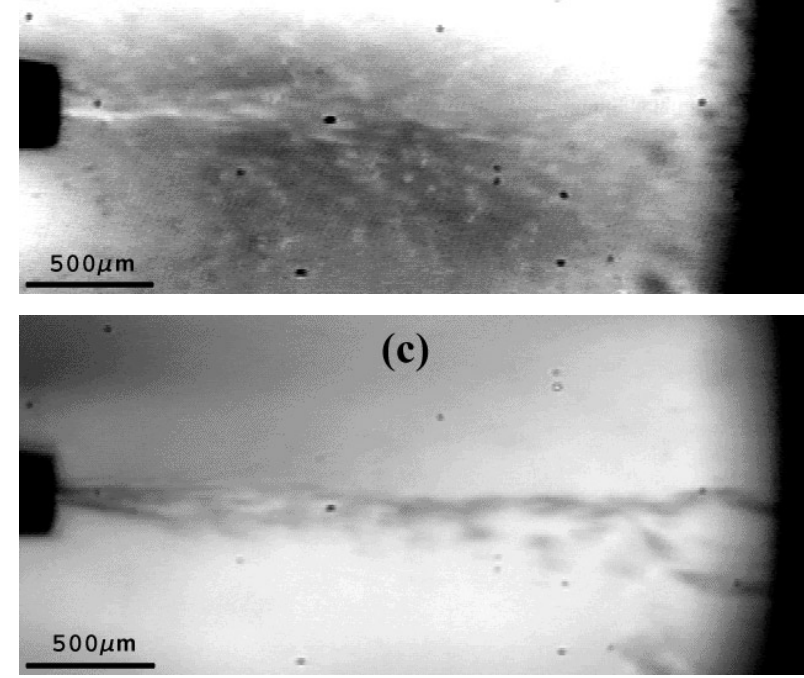

Figure 6. The effect of charging the Faraday cup. $T=2.2 \mathrm{~K}, \Delta P=5$ Torr and $V=2.5 \mathrm{kV}$. (a) No voltage is applied to the Faraday cup. The jet's surface is relatively smooth and there is no sign of deflected particles.

(b) $V_{F c}=+900 \mathrm{~V}$ is applied to the Faraday cup. The droplet fragments are deflected back and scattered.

(c) $V_{F c}=-900 \mathrm{~V}$ on the Faraday cup. The charged droplets are accelerated towards the cup and instabilities on the jet's surface are produced.

was observed when the discharge was monitored on the oscilloscope. By examining a large number of sequences, we estimated from the calculated flow rate that the pulse period was of the order of 1-30 $\mu$ s.

We were able to deflect the trajectories of the particles by applying a high-voltage potential $(V= \pm 900 \mathrm{~V})$ to the Faraday cup, which was located $0.7-1.0 \mathrm{~cm}$ from the needle's tip. Figure 6(a) shows an explosion with no voltage on the cap. Applying a positive potential to the Faraday cup reflected the particles, which were scattered within the droplet chamber (figure 6(b)). Negative potentials tended to destabilize the jet, by accelerating the jet towards the plate. This greater acceleration led to fluctuations on the surface of the jet (figure 6(c)).

A further increase in the needle potential, above the glow regime, increased the ion current until an arc occurred between the needle tip and the cap or nozzle mount. The arc led to a violent explosion of the liquid, followed by cessation of flow. The voltage had to be reduced before the flow of liquid helium could be re-established. The arc
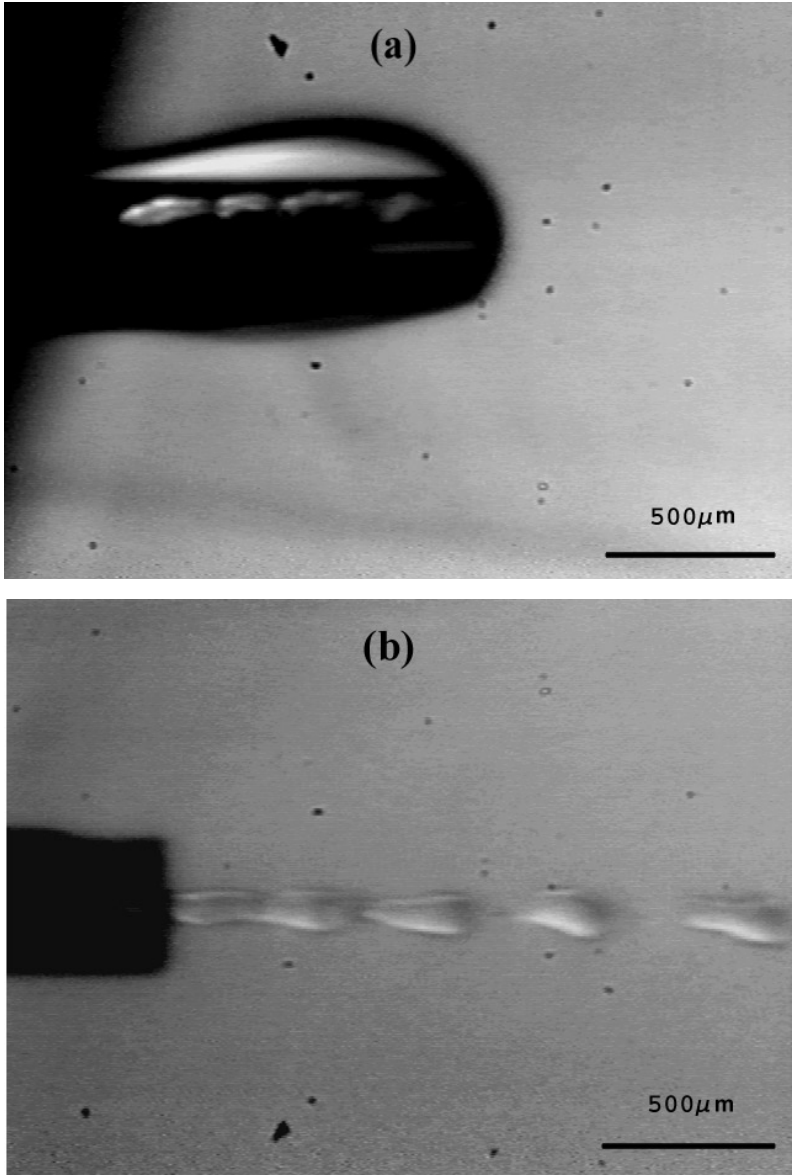

Figure 7. The formation of droplets in He II. $T=1.8 \mathrm{~K}$ and $\Delta P=-4$ Torr: (a) $V=0 \mathrm{~V}$ and (b) $V=1.75 \mathrm{kV}$. Large, irregularly shaped droplets $(200-300 \mu \mathrm{m})$ are formed.

was characterized by much higher currents (of the order of $10 \mu \mathrm{A})$ with large fluctuations of the order of $1 \mu \mathrm{A}$.

3.1.2. Temperature $3.5 \mathrm{~K}$. At $3.5 \mathrm{~K}$ and $\Delta P=5$ Torr, a higher threshold potential $(8-10 \mathrm{kV})$ was required in order to create the same sudden increase in ion current and shower-type explosion effects that were observed at the lower temperature of $2.2 \mathrm{~K}$. Several runs were performed at both temperatures to confirm that the increase in threshold voltage was not due to erosion of the needle tip.

3.1.3. Temperature $1.8 \mathrm{~K}$. At a source pressure of 11 Torr, the liquid helium equilibrium temperature is approximately $1.8 \mathrm{~K}$, which is about $0.4 \mathrm{~K}$ below the $\Lambda$ point of helium. We observed that liquid helium flowed out of the nozzle against a negative pressure gradient $(\Delta P=-4.0 \pm 0.5$ Torr) and wetted the glass surface with the voltage off (figure $7(\mathrm{a})$ ). Above $V \simeq 2 \mathrm{kV}$, the liquid was driven out of the nozzle in the form of large droplets $(r>100 \mu \mathrm{m})$. The droplets were irregular in shape (figure 7(b)), indicating that the droplets' surfaces were highly unstable in the presence of charges. In contrast to the experiments above $2.2 \mathrm{~K}$, no shower-explosion behaviour was observed at this temperature. 


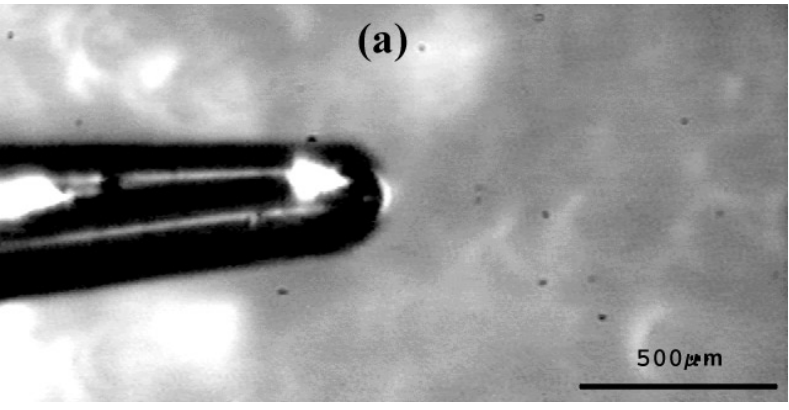

(b)
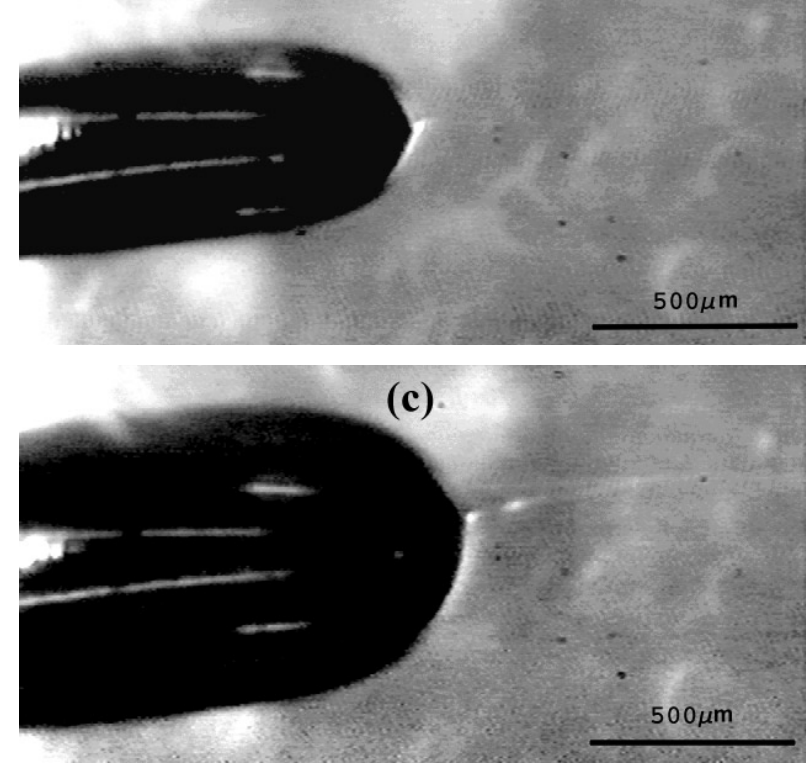

Figure 8. The effect of charge on liquid $\mathrm{N}_{2} . T=70 \mathrm{~K}$ and $\Delta P=20$ Torr. (a) $V=0 \mathrm{~V}$. Wetting of the capillary is observed. (b) $V=5 \mathrm{kV}$. The drop bound to the tip expands and a Taylor cone is seen. (c) $V=10 \mathrm{kV}$. A small jet is ejected.

\subsection{Liquid nitrogen}

The liquid nitrogen experiments were conducted at $T=$ $70 \pm 1 \mathrm{~K}$. Liquid nitrogen displayed a low-flow mode at small pressure differences $(\Delta P<25$ Torr $)$ and a jet mode at large pressure differences $(\Delta P>25$ Torr $)$.

3.2.1. Low-flow conditions. At pressure differences of 15-25 Torr across the nozzle, liquid nitrogen wetted the capillary when no voltage was applied, as depicted in figure 8(a). By applying a high voltage to the needle, a stable liquid nitrogen drop formed around the nozzle capillary (figure $8(\mathrm{~b})$ ). The drop induced by the electrostatic force increased in size as the applied voltage increased. Drops as large as $0.5 \mathrm{~mm}$ in radius were formed with needle voltages of $9 \mathrm{kV}$, above which fragmentation would occur. At voltages higher than $5 \mathrm{kV}$, the droplet started to distort by forming a wide conical tip and a protuberance appeared at the tip of the drop. Surface oscillations of the drop were observed, but were insufficient to cause fragmentation. Above $9 \mathrm{kV}$, depending on needle
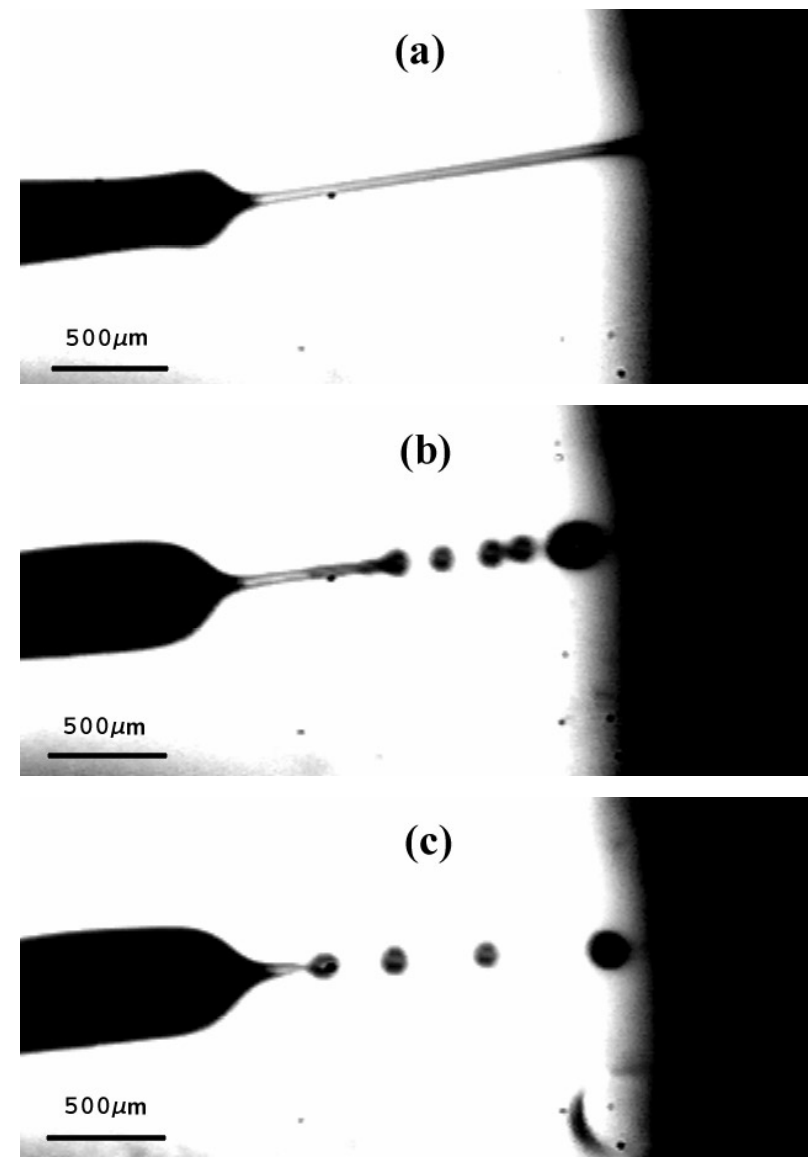

Figure 9. The effect of charge on spraying of liquid nitrogen at $T=70 \mathrm{~K}$ and $\Delta P=40$ Torr. (a) No voltage is applied to the needle. (b) $V=5 \mathrm{kV}$. (c) $V=8 \mathrm{kV}$. The jet's length is shortened and the droplets become slightly smaller as the voltage on the needle is increased.

conditions, the protuberance broke loose from the large stable drop to from a thin jet of liquid (figure 8(c)).

3.2.2. Jet flow. At pressure differences of $35-50$ Torr and $V=0-5 \mathrm{kV}$, a jet of liquid flowed from the nozzle. After a certain length the jet broke into a sequence of droplets with the jet length depending on the pressure difference (figure 9(a) depicts such a pressure-driven jet of liquid nitrogen, with the voltage off). At needle voltages higher than $5 \mathrm{kV}$, we observed the effects of charge-induced instability: the length for breakdown of the jet into droplets was shortened and the size of the droplets decreased (figures 9(b) and 9(c)), in accordance with the observations of Turnbull and co-workers [15]. A Taylor cone was clearly observable in front of the nozzle tip. Typically, the jet's diameter was about $50 \mu \mathrm{m}$ and the droplets, which broke off from the jet one at a time, had sizes ranging from 100 to $250 \mu \mathrm{m}$ in diameter, depending on the applied voltage. In contrast to the irregular shape of charged liquid helium II droplets, liquid nitrogen droplets were close to spherical. The current measured on the Faraday cup was approximately $3 \mathrm{nA}$. At very high voltages $(V>10 \mathrm{kV})$, arcing from the needle to the source body was observed. 
Table 1. A comparison of the charge per unit length for $\mathrm{He}$ and $\mathrm{N}_{2}\left(\mathrm{C} \mathrm{m}^{-1}\right)$ computed for the experimental source conditions (equation (2)), and the Rayleigh limits (equation (1)). Included are also the calculated rate of flow and velocity and some relevant physical properties [30,31].

\begin{tabular}{llllllcc}
\hline $\begin{array}{l}q / I_{\text {EXP }} \\
(\text { experimental) }\end{array}$ & $\begin{array}{l}q / I_{R L} \\
(\text { Rayleigh limit })\end{array}$ & $\begin{array}{l}\left(q / I_{\text {EXP }}\right) /\left(q / I_{R L}\right) \\
(\text { ratio })\end{array}$ & $\begin{array}{l}Q \\
\left(\mathrm{~m}^{3} \mathrm{~s}^{-1}\right)\end{array}$ & $\begin{array}{l}v \\
\left(\mathrm{~cm} \mathrm{~s}^{-1}\right)\end{array}$ & $\begin{array}{l}\text { Viscosity } \\
\left.(\mu \mathrm{Pa} \mathrm{s})^{-1}\right)\end{array}$ & $\begin{array}{l}\text { Surface tension } \\
\left(\mathrm{N} \mathrm{m}^{-1}\right)\end{array}$ \\
\hline $\mathrm{He}$ & $1 \times 10^{-7}$ & $2 \times 10^{-9}$ & 50 & $2.7 \times 10^{-9}$ & 70 & 3.85 & $3.301 \times 10^{-4}$ \\
$\mathrm{~N}_{2}$ & $9 \times 10^{-9}$ & $1 \times 10^{-8}$ & 1.1 & $3.1 \times 10^{-10}$ & 8.0 & 203.9 & $1.053 \times 10^{-2}$ \\
\hline
\end{tabular}

Arcing usually led to chaotic behaviour of the jet and to currents of several micro-amperes.

\section{Discussion}

\subsection{Electrostatic spraying}

The charging of liquid helium and nitrogen was evident when a voltage was applied to the needle. Even at relatively low voltages, wetting of the glass capillary was inhibited (most probably due to charging of the glass surface) and a 'stable' droplet formed on the nozzle's tip. At higher voltages, droplet formation was induced and measurable currents were collected for both liquids; however, the behaviours of helium and of nitrogen were different. In the case of helium, we observed pulsed explosions of liquid droplets or jets into showers of fine droplets. Charging of the droplet fragments was verified by observing their deflection upon applying a voltage potential to the collecting cap. The electrospraying of nitrogen was characterized by the formation of a Taylor cone at the nozzle tip. Instabilities of the cone led to the jet breaking up into singly charged particles several hundred micrometres downstream of the nozzle.

The instabilities of electrified jets and droplets were studied as early as 1882 by Lord Rayleigh [19]. By considering the balance of surface tension and Coulomb repulsion, Rayleigh derived an equation to predict the maximum charges sustainable on a droplet and on a cylindrical jet, above which instabilities lead to break-up. In the case of the cylindrical jet, Rayleigh obtained the following equation:

$$
(q / l)_{R L}=\left(6 \pi^{2} \gamma \varepsilon_{0} a\right)^{1 / 2}
$$

where $\gamma$ is the surface tension of the liquid, $\mathrm{a}$ is the diameter of the cylindrical jet, $\varepsilon_{0}$ is the electrical permittivity of the medium surrounding the jet and $(q / l)_{R L}$ is the Rayleigh charge limit per length of the jet. Empirically, it has been found that break-up of a charged cylindrical jet will occur at or below the Rayleigh limit [20].

In order to compare with the Rayleigh limit for liquid helium and liquid nitrogen under our experimental conditions, we had to estimate the charge per unit length. This quantity was predicted from the ratio of the observed current to the rate of flow:

$$
(q / l)_{\exp }=\frac{I}{Q} \frac{\pi a^{2}}{4} .
$$

While we measured the current $I$, we were unable to measure the liquid volumetric rate of flow $Q$ directly and we therefore computed the latter quantity. Because the dimensions of the capillary were narrow (the inner diameter of the capillary tapered from 450 to $70 \mu \mathrm{m}$ with a $50 \mu \mathrm{m}$ wire inside), viscous forces dominated flow. The estimated Reynolds number was of the order of $10^{-6}$, therefore we treated the flow as laminar. By modelling the nozzle as an annulus with an exponentially contracting outer wall (the glass inner diameter) and a straight inner wall (the needle's diameter), we were able to calculate the rate of flow numerically. The equation for the pressure drop of laminar flow in an annulus is

$$
\Delta P=\frac{8 \mu Q \Delta L}{\pi(R)^{4}}\left(1-\kappa^{4}-\frac{\left(1-\kappa^{2}\right)^{2}}{\ln \left(\kappa^{-1}\right)}\right)^{-1}
$$

where $\Delta P$ is the pressure drop across a length $\Delta L, \mu$ is the viscosity, $Q$ is the rate of flow, $R$ is the radius of the glass nozzle and $\kappa$ is the ratio of the outer wall diameter to the inner wall diameter. The flow equation was solved by differencing. In the numerical routine the nozzle was divided into infinitesimal sections and the pressure drop was calculated for each segment from equation (3). The pressure was summed over all sections and compared with the experimentally set pressure difference across the nozzle. The rate of flow was iterated until the total pressure drop converged to the experimentally measured pressure difference.

Results are given in table 1 . We find that in our experiments the liquid helium jets have charge densities about 50 times greater than the Rayleigh limit. In contrast, the experimental charge per length for the liquid nitrogen jets is comparable in magnitude to the computed Rayleigh limit. These results are consistent with, and provide an explanation for, the experimental observations. We would expect that any helium jet of dimension $100 \mu \mathrm{m}$ would undergo Coulomb explosion by fragmenting into many small droplets and that a Taylor cone would not be observed. On the other hand, the surface charging of liquid nitrogen jets is of the critical magnitude to affect the stability of a jet; thus, we would expect to observe droplets formed from Rayleigh-Taylor instabilities.

In the case of liquid nitrogen, the greater surface tension of the liquid (see table 1) balances the repulsion between the surface charges, allowing the formation of a stable drop of order $0.5 \mathrm{~mm}$ on the nozzle's tip. By increasing the voltage one can visually observe the propagation of instabilities on the liquid's surface, the formation of a protuberance and the breakage of the jet into droplets.

The Rayleigh limit as calculated above is a simple model for the charge of a stationary cylinder. It does not account for the stabilization or destabilization effects 
induced by the acceleration of the jet due to the electrical field of the needle or fluid motion. Turnbull [21] has studied surface instabilities of electrified jets of insulated liquids with a more detailed model. He introduces a first-order perturbation onto the equations of motion, continuity and electrical potential for an equilibrated liquid jet in order to simulate jet instabilities (the rate of growth of perturbations) as a function of the wavelength of the surface oscillations. Turnbull identifies the charge density on the surface of the jet and the presence of a tangential field along the jet, due to the electrical field of the needle, as the main effects leading to stabilization or destabilization of the jet. For short-wavelength oscillations $(\lambda \simeq 2 \pi r$, where $r$ is the jet's radius), which we observe in our nitrogen jets, the model developed by Turnbull indicates that the tangential electrical field tends to stabilize the jet's surface whereas the surface charge destabilizes the jet. Taken together, both surface charging and tangential fields stabilize shortwavelength oscillations because they produce a shear force in the direction of the jet. In contrast, our observations show that, on applying a voltage to the jet, there is a reduction in length of the jet. This indicates that we are in a regime under which destabilization of the jet by the surface charge is more effective than is stabilization by the tangential field.

In the case of liquid helium, since the charge density far exceeds the Raleigh limit, explosions occur at the point of charge injection. A long-wavelength $(\lambda \geq r)$ hydrodynamic analysis of jet instabilities such as Turnbull's is no longer valid. The Coulomb repulsion effect dominates the surface tension term and leads to explosion of the jet into several smaller droplets $(r<10 \mu \mathrm{m})$. Two main factors contribute to the dramatic differences between helium and nitrogen: the very low surface tension of liquid helium, about two orders of magnitude smaller than that of nitrogen, and the higher current observed in helium, about two orders of magnitude larger than that observed in nitrogen. The heat dissipated into the jet may also play a role in the abrupt explosion of helium. We estimate an upper limit on the average electrical power dissipated at the needle of $I V \simeq 0.4 \mathrm{~mW}$, which is slightly less than the energy necessary to vaporize liquid helium.

The Coulomb explosions we observe in helium are of a regular pulsed nature. However, there is no evidence of pulsing behaviour from the waveform of the high voltage supplied to the needle. We also observe glowing at the needle and on one or more points on the glass tip while ionization takes place. These observations suggest that ionization may be occurring both at the needle tip and at charged points on the glass surface.

\subsection{The field ionization mechanism of charging}

Field emission and field ionization of cryogenic liquids ( $\mathrm{He}, \mathrm{H}_{2}, \mathrm{~N}_{2}, \mathrm{O}_{2}$ and $\mathrm{Ar}$ ) have been studied by Gomer and Halpern using a point-to-plane field emission tube $[12,13]$. Our results were similar to those obtained by Gomer and Halpern in the existence of a sharp onset ( $V=1.3 \mathrm{~V}^{-1}$ in Gomer's work) at which currents of the order of micro-amperes set in. They also observed selfsustaining discharges when the voltage was reduced until a sharp cut-off ( $V=0.64 \mathrm{~V}^{-1}$ in Gomer's work).

The electrical breakdown of liquid helium [22-24] and other cryogenic liquids [25,26] has been studied extensively by research groups interested in the dielectric properties of helium and other cryogenic liquids as insulators. In these studies, however, little attention was given to the mechanism of ionization.

4.2.1. Higher currents in liquid helium. The model presented by Gomer and Halpern describes field ionization in terms of tunnelling of electrons from the atoms to the electrode. In their experiments, they observed currents from discharges in liquid helium higher than those for other cryogenic liquids. They explained the higher current in liquid helium in terms of the possibility of ionizing avalanches, the higher mobility of ions in liquid helium and the formation of gas bubbles.

The greater probability of charge multiplication (avalanching) presents an explanation for the higher current in helium. Charge multiplication can take place in the liquid through ion impact ionization when the ions are allowed to obtain enough translational energy before colliding. In liquid nitrogen, some kinetic energy of the accelerated ions can be converted into vibrational and rotational modes of the liquid molecules through inelastic collisions. This energy conversion mechanism impedes the ions reaching sufficient kinetic energies for ion impact ionization within one mean free path. Helium ions, on the other hand, are much hotter because of the lack of energy quenching mechanisms. The higher current in helium can also be explained in terms of the ion mobility. Gomer measured mobilities of helium and nitrogen ions and found that the helium ion had a mobility ten times higher than that of the nitrogen ion [27].

The lower heat of vaporization of helium (about 50 times lower than for nitrogen) can also be related to the higher current measured in helium. The energy associated with the hot ions can create low-density bubble sites. Avalanching followed by discharging occurs when the bubbles grow to a certain size limit, at which the free length is sufficient for ions to create multiple charging. Since the heat of vaporization is lower, formation of bubbles in liquid helium is more likely.

4.2.2. The temperature dependence. The temperature dependence of helium I discharges can be understood in terms of the bubble mechanism. Gerhold, in his study of dielectric breakdown of cryogenic gases and liquids, has pointed out that, during bubble formation, the dielectric strength decreases rapidly from that of the liquid to that of the vapour until the Paschen minimum is reached, whereupon breakdown takes place. The discharge inside a bubble is therefore similar to a gas phase discharge. The dielectric strength of saturated helium vapour increases by a factor of about seven on going from 2.2 to $3.5 \mathrm{~K}[22,28]$. This change in dielectric strength may in part explain why the threshold voltage is higher at $3.5 \mathrm{~K}$ than it is at $2.2 \mathrm{~K}$. 


\subsection{Superfluid helium}

We have obtained preliminary results on the charging of superfluid helium at $1.8 \mathrm{~K}$ flowing against small negative pressure gradients. The behaviour of superfluid helium differs markedly from that of normal helium at $2.2 \mathrm{~K}$. In the absence of an electrical field, the flow of superfluid helium through the capillary against a pressure gradient is a manifestation of the well known 'fountain effect' of helium II. This mass transport results from the flow of the Bose condensate to compensate for entropy differences between thermodynamic states of the liquids on each side of the capillary. This superleak of helium II continuously drives liquid out of nozzle until the chemical potentials are balanced across the nozzle. Since the surface tension is low for superfluid helium, we observe wetting on the capillary's exterior and the formation of large, stationary drops on the capillary's tip.

With modest electrical potentials ( $2 \mathrm{kV}$ on the needle), a jet emerges due to the presence of charges on the jet and the electric field from the needle. We observe hydrodynamic Rayleigh-Taylor instabilities leading to the formation of large droplets $(r>100 \mu \mathrm{m})$ of irregular shape, in contrast to the Coulomb explosions and formation of fine particles seen at $2.2 \mathrm{~K}$.

The observation of Rayleigh-Taylor instabilities instead of Coulomb explosion suggests that the surface charge density in the present case is much lower than that in the case of jets of normal liquid helium, despite the fact that field ionization currents tend to be higher at lower temperatures. This qualitative difference can readily be explained if the helium is below the superfluid transition. In this case, the superfluid component of the helium II will exhibit inviscid flow. The 'superleak' through the capillary leads to rates of liquid flow that can be orders of magnitude greater than the viscosity-limited flow of normal helium. The higher rates of flow would significantly reduce surface charge densities for a given current. However, we did not measure the current in these preliminary experiments and therefore cannot estimate the surface charge densities. The irregular shape of the droplets is also evidence for superfluidity. Helium II is characterized by a lower surface tension, which leads to the irregular shape of the droplets in the jet. Further experiments are under way elsewhere.

\section{Conclusion}

We have generated positively charged droplets by field ionization of liquid helium using an electrostatic spraying apparatus. A comparison of these results with those on liquid nitrogen indicates a qualitative difference in the respective mechanisms of droplet formation. We have found that electrostatic spraying of liquid helium at 2.2 $\mathrm{K}$ results in a Coulomb explosion forming a spray of fine droplets with currents of $0.1-0.4 \mu \mathrm{A}$, two orders of magnitude greater than currents detected for liquid nitrogen. Electrical breakdown occurs at about $2 \mathrm{kV}$ for helium, significantly less than the voltages $(\approx 8 \mathrm{kV})$ required for liquid nitrogen. Ionization of liquid nitrogen, like that of insulating cryogenic liquids such as hydrogen and argon, exhibits classic Rayleigh-Taylor instability. Charging leads to the formation of a Taylor cone or jet; droplets with dimensions similar to the jet are formed sequentially downstream by instabilities.

The difference between the behaviours of liquid helium and liquid nitrogen lies in the much higher currents obtained from field ionization in liquid helium. The resulting surface charge density greatly exceeds the Rayleigh limit for jets of dimension $a \approx 100 \mu \mathrm{m}$. The observed Coulomb explosions in liquid helium are a direct consequence of the excess in surface charge density.

\section{Acknowledgments}

This work was supported by American Technologies Group, Inc. We would like to thank Gilbert Wong for his many technical contributions.

\section{References}

[1] Williams G A and Salomaa M M 1984 J. Low Temp. Phys. 57539

[2] Salomaa M M and Williams G A 1981 Phys. Rev. Lett. 47 1730

[3] Akinci G and Northby J A 1979 Phys. Rev. Lett. 42573

[4] Hautojarvi P, Rytsölä K, Tuovinen P, Vehanen A and Jauho P 1977 Phys. Rev. Lett. 38842

[5] Gspann J and Vollmar H 1981 J. Low Temp. Phys. 45343

[6] Buchenau H, Toennies J P and Northby J A 1991 J. Chem. Phys. 958134

[7] Northby J A, Kim C and Jiang T 1994 Physica B 197426

[8] Joppien M, Muller R and Möller T 1993 Z. Phys. D 26382

[9] Fröchtenicht R, Henne U, Toennies J P, Ding A, Fieber-Erdmann M and Drewello T 1996 J. Chem. Phys. 1042548

[10] Boyle F P and Dahm A J 1976 J. Low Temp. Phys. 23477

[11] Volodin A P and Khaikin M S 1979 JETP Lett. 30572

[12] Halpern B and Gomer R 1969 J. Chem. Phys. 511048

[13] Halpern B and Gomer R 1969 J. Chem. Phys. 511031

[14] Halpern B and Gomer R 1969 J. Chem. Phys. 515709

[15] Kim K and Turnbull R J 1976 J. Appl. Phys. 471964

[16] Woosley J P, Turnbull R J and Kim K 1982 IEEE Trans. Indust. Appl. 18314

[17] Woosley J P, Turnbull R J and Kim K 1988 J. Appl. Phys. 644278

[18] Woosley J P and Turnbull R J 1977 Rev. Sci. Instrum. 48 254

[19] Rayleigh L 1882 Phil. Mag. 14184

[20] When $Q / l$ exceeds the Rayleigh limit, breakdown or Coulomb explosion occurs

[21] Turnbull R J 1992 IEEE Trans. Indust. Appl. 281432

[22] Gerhold J 1972 Cryogenics 12370

[23] Gerhold J 1989 IEEE Trans. Electr. Insulators 24155

[24] Blank C and Edwards M H 1960 Phys. Rev. 11950

[25] Gerhold J 1979 Cryogenics 19571

[26] Jones H M and Kunhardt E E 1995 J. Phys. D: Appl. Phys. 28178

[27] Gomer R 1972 Acc. Chem. Res. 541

[28] Keller W 1955 Phys. Rev. 971

[29] William E K 1969 Helium-3 and Helium-4 (New York: Plenum)

[30] Lide D R 1990-91 CRC Handbook of Chemistry and Physics 71st edn (Boston Raton, FL: CRC) 